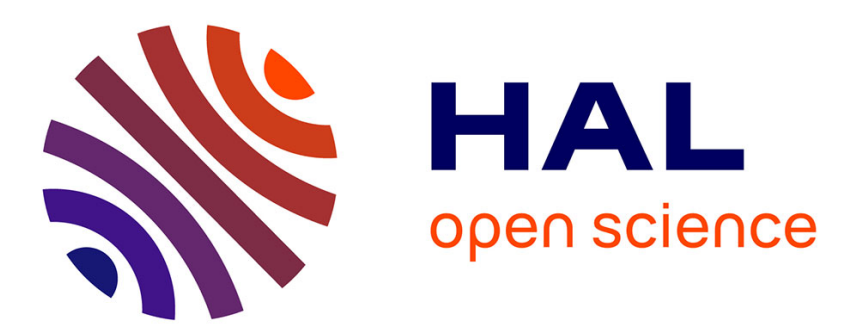

\title{
Fusion hindrance and synthesis of superheavy elements
}

Y. Abe, G. I. Kosenko, C. W. Shen, B. Bouriquet, A. Marchix, D. Boilley, B.

Giraud

\section{To cite this version:}

Y. Abe, G. I. Kosenko, C. W. Shen, B. Bouriquet, A. Marchix, et al.. Fusion hindrance and synthesis of superheavy elements. Physics of Atomic Nuclei / Yadernaya Fizika, 2006, 69, pp.1101-1109. 10.1134/S1063778806070027 . in2p3-00087646

\section{HAL Id: in2p3-00087646 https://hal.in2p3.fr/in2p3-00087646}

Submitted on 25 May 2021

HAL is a multi-disciplinary open access archive for the deposit and dissemination of scientific research documents, whether they are published or not. The documents may come from teaching and research institutions in France or abroad, or from public or private research centers.
L'archive ouverte pluridisciplinaire HAL, est destinée au dépôt et à la diffusion de documents scientifiques de niveau recherche, publiés ou non, émanant des établissements d'enseignement et de recherche français ou étrangers, des laboratoires publics ou privés. 

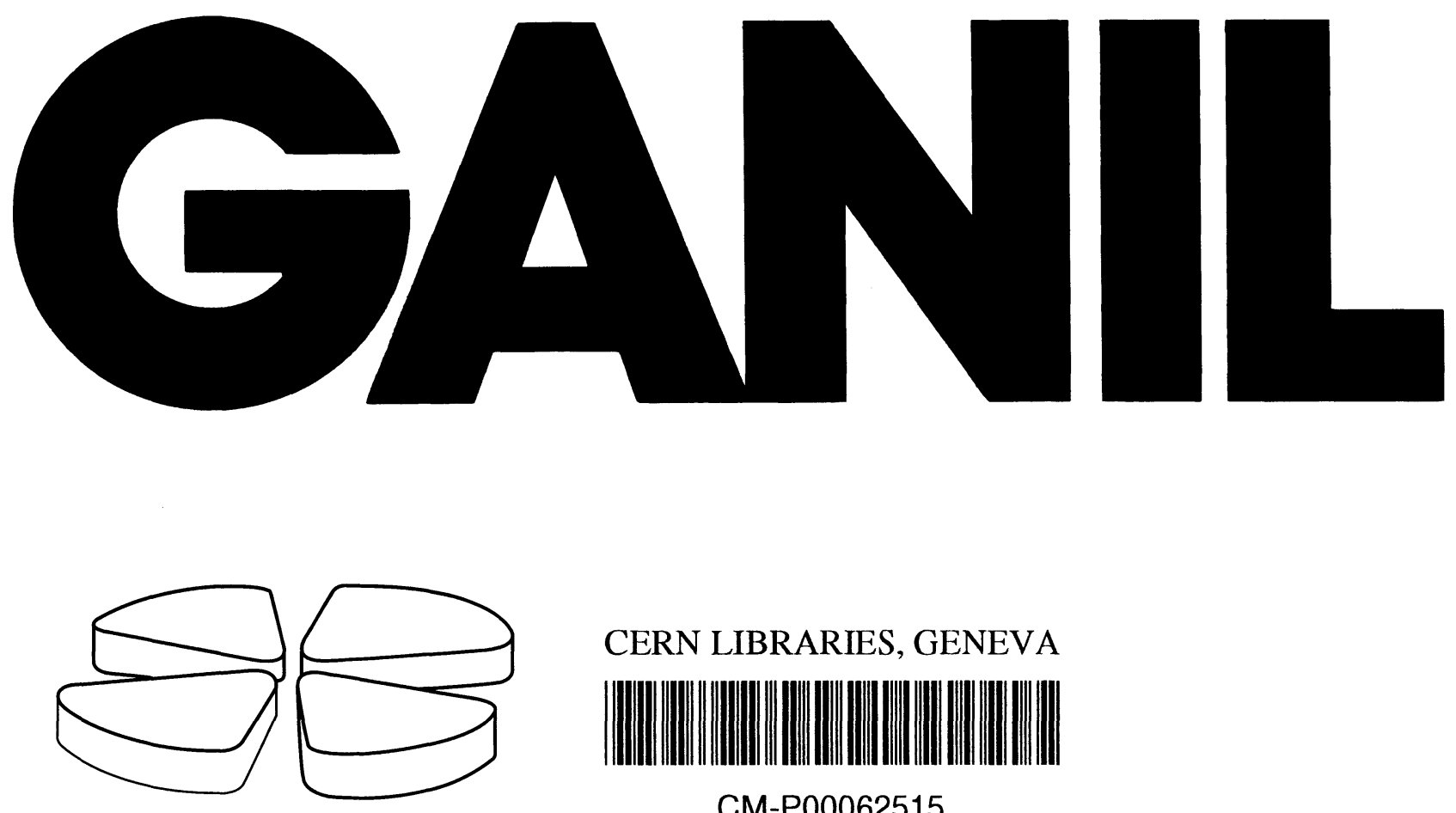

CERN LIBRARIES, GENEVA

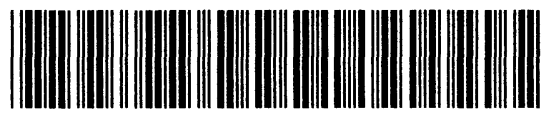

CM-P00062515

FUSION HINDRANCE and SYNTHESIS of SUPERHEAVY ELEMENTS

\footnotetext{
Y. Abe ${ }^{1}$, G. Kosenko², C.W. Shen ${ }^{3}$, B. Bouriquet ${ }^{4}$ A.Marchix ${ }^{5}$, D. Boilley ${ }^{5}$, B. Giraud ${ }^{6}$

${ }^{1}$ Research Center for Nuclear Physics, Osaka University, Ibaraki, Japan

2 Physics Department, Omsk University, Omsk, Russia

${ }^{3}$ School of Science, Huzhou Teachers College, Huzhou 313000, China

${ }^{4}$ Department of Nuclear Physics, Australian National University, Canberra, Australia

${ }^{5}$ Ganil, BP 55027, 14076 Caen cedex 05, France

${ }^{6}$ Service de Physique Theorique, CEN-Saclay, Gif/Yvette, France
}

Presented at the 55 International Meeting on Nuclear Spectroscopy and Nuclear Structure

"Frontiers in the Physics of Nuclei »

Russia, St. Petersburg, Peterhof, June 28-July 01, 2005

CERN LIBRARIES, GENEVA 


\title{
FUSION HINDRANCE and SYNTHESIS of SUPERHEAVY ELEMENTS
}

\author{
Y. Abe ${ }^{1}$, G. Kosenko ${ }^{2}$, C.W. Shen ${ }^{3}$, B. Bouriquet ${ }^{4}$ A.Marchix $^{5}$, D. Boilley ${ }^{5}$, B. Giraud ${ }^{6}$ \\ ${ }^{1}$ Research Center for Nuclear Physics, Osaka University, Ibaraki, Japan \\ ${ }^{2}$ Physics Department, Omsk University, Omsk, Russia \\ ${ }^{3}$ School of Science, Huzhou Teachers College, Huzhou 313000, China \\ ${ }^{4}$ Department of Nuclear Physics, Australian National University, Canberra, Australia \\ ${ }^{5}$ Ganil, BP 55027, 14076 Caen cedex 05, France \\ ${ }^{6}$ Service de Physique Theorique, CEN-Saclay, Gif/Yvette, France
}

\section{Introduction}

Experimental efforts [1] have been devoted over several decades for syntheses of the superheavy elements whose existence is predicted by microscopic theories of nuclear structure [2] in terms of a possible existence of a double magic nucleus after ${ }^{208} \mathrm{~Pb}$ or of a generalized concept of the shell correction energy [3]. But since there had been no reliable reaction theory for the synthesis, the experiments are based on systematics of the available data so far, taking into account reaction Q-values etc. But unexpectedly, fusion hindrance were observed and its reaction mechanism had not yet been understood well so long [4], although it is crucially important for the synthesis. In order to clarify the mechanism and to provide the theoretical framework for calculation of fusion probability, the present authors et al. have recently proposed the new two-step model for fusion of massive heavy ion systems [5]. There, the fusion process is divided into two sequential steps, i.e., the approaching phase of passing over the Coulomb barrier and the formation phase of the spherical compound nucleus. Thus, the fusion probability $P_{\text {fusion }}$ is given by the product of the sticking probability $P_{\text {stick }}$ for the first step and the formation probability $P_{\text {form }}$ for the second step.

$$
P_{\text {fusion }}^{J}\left(E_{\text {c.m. }}\right)=P_{\text {stick }}^{J} \cdot P_{\text {form }}^{J},
$$

where $J$ and $E_{\text {c.m. }}$. denotes the total angular momentum of the system and the incident kinetic energy in the c.m. system.

In both phases, dissipation of the collective energy and its associated fluctuation play an essential role, as is explained in the next section.

In addition to the fusion hindrance, another difficulty in synthesis of the superheavy elements stems from the fragility of the nuclei that their stability is given solely by the so-called shell correction energy, because of no fission barrier in the Liquid Drop Model (LDM) for nuclei with fissility about 1 [6]. Naturally, compound nuclei formed by the fusion reaction are excited, which means that the shells are more or less destroyed. Thus, the shell correction energies, which stabilise the nuclei, are diminished. Survival probability $P_{\text {surv }}$ of the compound nuclei against fission decay is calculated by the competition between fission decay with barrier depending on excitation energy and neutron emission which makes the compound nuclei to cool down and to restore the shell correction energies. This process is, as usual, treated by the statistical theory of decay, where the absolute values of the shell correction energies and their temperature dependence play a decisive role in determination of the survival probability. They are properly taking into account through the level density parameter in the statistical code [7]. Residue cross sections are, of course, given by the product of the fusion and the survival probabilities.

$$
\sigma_{r e s}\left(E_{e . m .}\right)=\pi \cdot \lambda^{2} \sum_{J}(2 J+1) \cdot P_{\text {fusion }}^{J}\left(E_{c . m .}\right) \cdot P_{\text {surv }}^{J}\left(E^{*}\right),
$$

where $\lambda$ denotes the de-Broglie wave length divided by $2 \pi$, and the excitation energy $E^{*}=E_{c . m .}+Q$ with the reaction $Q$-value. 
Examples of excitation functions for residues will be given in section 3 . In section 4, discussion will be given on attempts for how to reach $N=184$ closed shell, using neutron-rich secondary beams.

\section{Two-Step Model of Fusion of Massive Systems}

In lighter heavy ion systems, targets and projectiles are expected to fuse, once they pass through the Coulomb barrier to contact with each other. But it is not always the case in massive systems with the fissility of the compound nuclei near 1 . This is simply understood by the property of LDM energy. Fig. 1 schematically shows the LDM potential surface as a function of the elongation (distance between two mass centers in two-center parametrization) and the mass-asymmetry parameters. Since the fissility is nearly equal to 1 , the saddle point is near the spherical shape. On the other hand, the contact configuration (di-nucleus configuration) with the mass-symmetric entrance channel is apparently far outside from the spherical shape with the lower LDM energy, which means that even if the target and the projectile contact to form the di-nucleus configuration after overcoming the Coulomb barrier, the system does not automatically fuse, but is under driving force back to re-separation due to the potential slope. In order to form the spherical compound nucleus, the amalgamated system has to climb up over the saddle point. This is an essential difference from lighter systems and is a basic cause of the hindrance [8]. As an example with rather small mass-asymmetry, ${ }^{86} \mathrm{Kr}+{ }^{208} \mathrm{~Pb}$ system is shown. It is readily seen that the system has to climb up to overcome a conditional saddle point on the ridgeline. For a case with larger mass-asymmetry, the relative height of the conditional saddle point measured from the contact configuration is much smaller, i.e., the hindrance is expected to be weaker than the mass-symmetric case. As an example with large asymmetry, ${ }^{48} \mathrm{Ca}+{ }^{244} \mathrm{Pu}$ system is also shown in the figure, which has almost no relative saddle height. Even more is that the relative heights are smaller as the fissility of the compound nucleus is smaller, because the saddle point or the ridgeline locates outside the spherical shape and closer to the contact configurations. These are in accord with the observations on the hindrance. For quantitative study of the fusion probability, we have to take into account also the sticking probability of the approaching phase.

\subsection{Approaching phase : over Coulomb barrier}

As is well-known in the Deep-Inelastic Collisions (DIC) [9], interactions between heavy ions are strongly dissipative. Therefore, we have to take into account the aspect also in the fusion reaction. Although quantum effects are expected in penetration of the Coulomb barrier [10], we adopt a classical mechanics for trajectory with the surface friction model (SFM) [11] for the sake of the consistency with the treatments in DIC. The model, however, is applied up to the contact point of the target and the projectile, thereafter, the system is treated as an amalgamated mono-nucleus, which is discussed in the next subsection. Furthermore, consistently with the dissipation-fluctuation theorem [12], we introduce the associated fluctuation to the friction, i.e., the associated random force in the equation. Thus, the equation for trajectory is now a Langevin equation. We, thus, have to calculate an ensemble of trajectories. Since the details of the model are already given elsewhere [13], here we summarise essential results of the model at the contact point;

1. The incident kinetic energy is completely dissipated, i.e., the mean value of the radial momentum is equal to zero (note that in lighter systems it is not the case),

2. The distribution of the radial momentum is approximately Gaussian with the variance consistent with the temperature of the intrinsic nucleonic degrees of freedom whose excitation energy is transferred from the kinetic energy due to the friction 


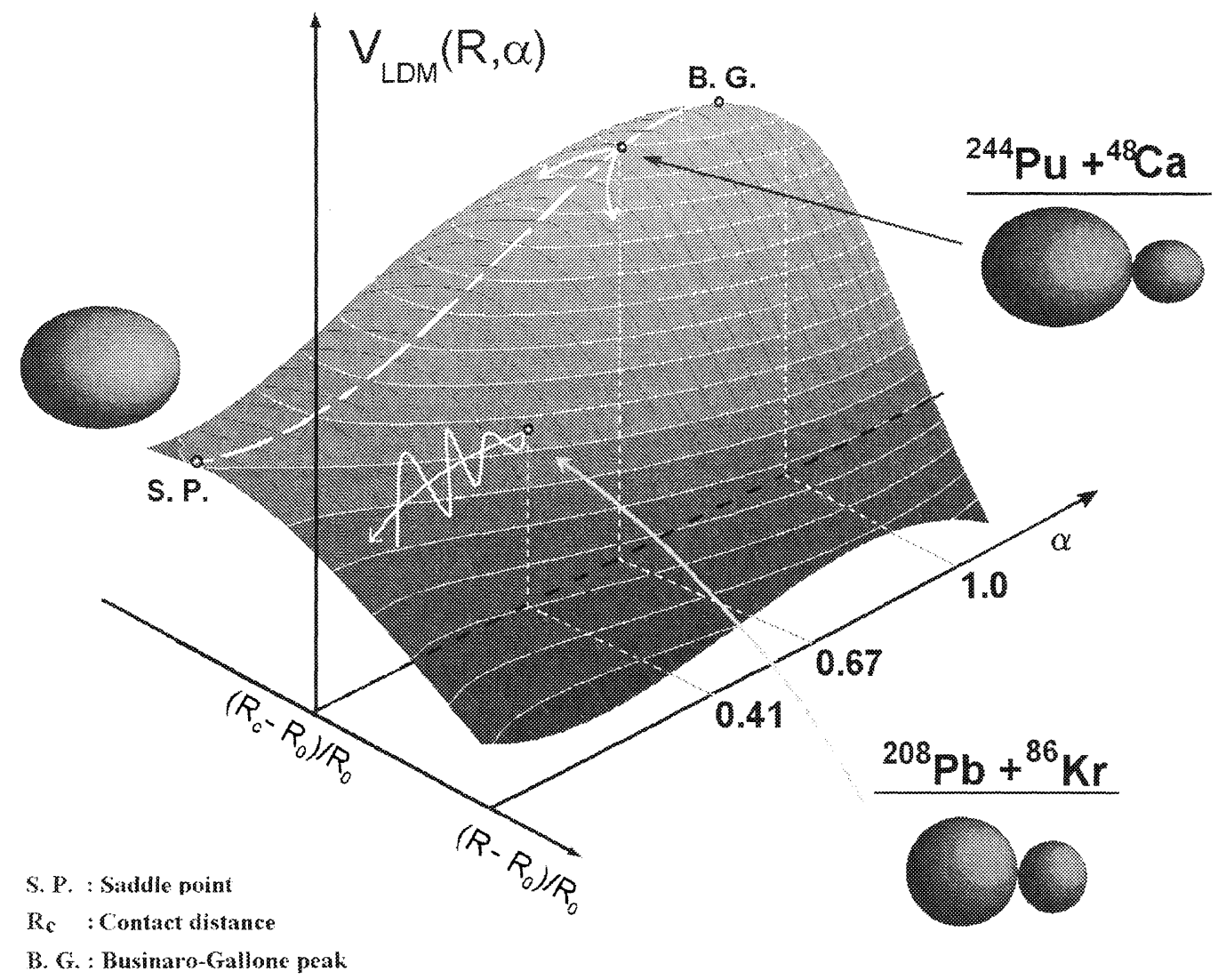

Figure 1: LDM energy surface in the two-dimensional space of the distance $\mathrm{R}$ and the mass-asymmetry $\alpha$ for the fissility being about 1. Rc denotes the distance between the centers of two spheres corresponding to the projectile and the target of the incident channel at the contact configuration, while $R_{0}$ does that between the mass centers of the semi-spheres corresponding to those at the compound nucleus limit.

force. In other words, the distribution is that of the thermal equilibrium.

3. Energy dependence of $\mathrm{P}_{\text {stick }}$ is similar to the transmission coefficient of the Coulomb barrier, but is much slower in increase as $\mathrm{E}_{c . m}$. increases.

Thus, the distribution of the momentum $S$ obtained with SFM generalized to include the fluctuation is approximately expressed as follows,

$$
S^{J}(p)=P_{\text {stick }}^{J}\left(E_{c . m .}\right) \cdot g^{J}(p), \quad g^{J}(p)=\frac{1}{\sqrt{2 \pi \mu T}} \exp \left[-\frac{(p-<p>)^{2}}{2 \mu T}\right],
$$

where $\langle p\rangle$ denotes the average value of remaining momenta that is almost equal to zero in massive systems calculated. $T$ denotes the temperature of the compound system with the di-nucleus configuration. $\mu$ is the reduce mass. The point 1 means, for the second phase of shape evolution, that the average trajectory has no initial impulse toward the inner direction at the contact configuration, irrespectively of incident kinetic energy and thus, is not capable to overcome the conditional saddle or the ridgeline! The point 2, however, indicates that there is a small probability for overcoming the saddle from the tail part of the distribution which has large innerward radial momenta. Of course, this is simply about the initial condition for the second phase, while the dissipative dynamics in the second phase itself gives rise to an extreme reduction of a probability for forming the spherical shape, as is discussed below. The point 3 appears to already explain the hindrance partially. 


\subsection{Formation phase : over conditional saddle point}

As is discussed in the previous subsection, there is the conditional saddle point or the ridgeline between the contact configuration and the spherical compound nucleus, which has to be overcome for formation of the compound nucleus, starting from the point on the potential slope, i.e., under action of the driving force back to re-separation. Furthermore, the system is excited with the energy transferred from the incident kinetic energy due to the friction, which means that there is a thermal agitation to the collective shape motions by incessant interactions with the thermal motion of nucleons, i.e., with the heat bath. This is treated, as usual, in terms of frictional force as an average effect and of the remaining rapidly fluctuating force. Therefore, the dynamics of the shape evolution has to be solved again with a Langevin equation with a multi-dimensional potential landscape like that shown in Fig. 1, and with a frictional force and its associated random force [12]. As a frictional force for the shape motions, we adopt so-called one-body model, i.e., the wall-and-window formula(WWF) [14], according to our experiences in analyses of anomalous multiplicities of pre-scission neutrons [15]. As stated above, an initial value for the radial momentum is given as the thermal distribution obtained as the results of the approaching phase. This gives a new connection method between the sequential steps of the two-body collision process and the one-body shape evolution of the amalgamated system. The method is completely different from adiabatic or from diabatic, but should be called a statistical connection.

\subsection{Mechanism of fusion hindrance}

The hindrance mechanism had been waited for so long to be clarified. Thus, before proceeding to the realistic calculations, it is meaningful to remind the recent accomplishments on underlying mechanism for the fusion hindrance [16]. To facilitate analytic study of the fusion mechanism, we employ a simple one-dimensional model for overcoming of a conditional saddle point, under a friction and its associated fluctuation. Approximating the shape of the saddle point with an inverted parabola and the frictional force with a constant friction coefficient $\gamma$, the equation becomes as follows,

$$
\frac{d p}{d t}=\mu \cdot \omega \cdot q-\beta \cdot p+R(t),
$$

where $\mu$ and $\omega$ denote the inertia mass for the radial motion and the frequency of the parabola, while $\beta$ and $R(t)$ do the reduced friction coefficient $\gamma / \mu$ and the fluctuating force assumed to be Gaussion, satisfying the dissipation-fluctuation theorem, i.e.,

$$
<R(t) \cdot R\left(t^{\prime}\right)>=2 \cdot \gamma \cdot T \cdot \delta\left(t-t^{\prime}\right),
$$

where the memory effect is neglected and $T$ denotes the temperature of the heat bath, i.e., that of the compound nucleus. The symbol $<>$ denotes the average over all the possible realizations of $R(t)$. With solutions of Eq.(4), the formation probability $F$, i.e., the probability for entering into the other side of the parabola with a given initial momentum $p_{0}$ is simply given by the error function as follows,

$$
F\left(p_{0}\right)=\frac{1}{2} \operatorname{erfc}\left[\sqrt{\frac{\eta+\eta^{\prime}}{2 \eta}}\left[\sqrt{\frac{B}{T}}-\frac{1}{\eta+\eta^{\prime}} \sqrt{\frac{K}{T}}\right]\right],
$$

where $B=\mu \cdot \omega^{2} \cdot q_{0}^{2} / 2$ with $q_{0}$ being the contact distance denotes the relative height of the saddle point measured from the contact configuration and $K$ denotes the initial kinetic energy $p_{0}^{2} / 2 \mu$ at the contact point. The non-dimensional parameter $\eta$ is defined as $\mu / 2 \omega$ and $\eta^{\prime}$ is $\sqrt{1+\eta^{2}}$. Here, it is interesting to notice that Eq.(6) gives a simple explanation 
of the so-called extra-push energy. In order for the probability $F$ to be equal to $1 / 2$, the argument of the error function must be zero. Therefore, the required initial kinetic energy $K$ should be equal to $\left(\eta+\eta^{\prime}\right)^{2} \cdot B$. Since the parameter $\eta$ is a few to several with WWF, the factor in front of $B$ can be easily equal to several to ten. Thus, even if the height of the saddle point $\mathrm{B}$ in LDM is small, say, a few $\mathrm{MeV}$, the effective height becomes a few tens $\mathrm{MeV}$, which is required for the kinetic energy. Consequently, the probability $F$ becomes extremely small in low energies. Thus, this is considered to be a basic mechanism for the fusion hindrance. Furthermore, the kinetic energy $K$ is that at the contact point, which is much smaller than that of the incident energy, because the incident energy is dissipated during the approaching phase up to the contact point. Actually, the mean value is nearly zero in massive systems, as is given in the previous subsection.

In order to obtain the formation probability for a given incident energy, we have to make an average of the probability $F$ given in Eq.(6) over the distribution of the momentum at the contact point calculated for the incident energy $E_{c . m}$. According to our experiences in massive systems, the distribution is that of the thermal equilibrium, as state in the point 2 in the previous subsection. The result is again given by the error function,

$$
P_{\text {form }}^{J}=\frac{1}{2} \operatorname{erfc}\left[\sqrt{\frac{B}{T}}\right] .
$$

As discussed by Swiatecki et al. [17], this is the solution of Smoluchowski equation corresponding to the overdamping limit of Kramers equation, or of Langevin equation. (Generally, the argument of the error function in Eq.(7) includes the remaining momentum which comes from $\langle p\rangle$ in Eq.(3). )

Of course, the fusion probability is given by the product of the sticking probability and the formation probability, as given in Eq.(1). Thus, it is clear that the fusion probability is determined by the combination of the entrance channel parameters and the compound nucleus parameters. With the aid of the analytic formula given in Eq.(7), a careful analysis would give a new order parameter controlling the hindrance, such as a combination of the effective fissility parameter of the entrance channel $x_{\text {eff }}$ and the fissility parameter of the compound nucleus $x$. In other words, the present model provides a global understanding of the hindrance mechanism which had not been clarified. And furthermore, it naturally provides the dynamical method to calculate fusion probability of massive heavy ion systems, which is necessary for prediction of synthesis of the superheavy elements.

\section{Fusion Excitation Functions and Residue Cross Sections for the Superheavy Elements}

Realistic calculations for the formation probability are made in two-dimensional collective space with the radial distance and the mass-asymmetry such as shown in Fig.1, and with the hydrodynamical inertia mass and the WWF for the friction. It would be better to include one more degree of freedom, such as the necking parameter, but effects would not be so large in fusion process, considering the fact that to fuse or not to fuse is determined rather quickly. (see Ref. [16]).

\subsection{Fusion probability and fusion excitation functions [18]}

According to the present model, we calculate sticking and formation probabilities, and then fusion probabilities by their products. In Fig. 2, an example of them is shown for the $s$-wave of ${ }^{58} \mathrm{Fe}+{ }^{208} \mathrm{~Pb}$ system. The abscissa is $\mathrm{E}_{c . m}$., while the ordinates are the probabilities (note the different scales among the panels). It is readily seen that the fusion 

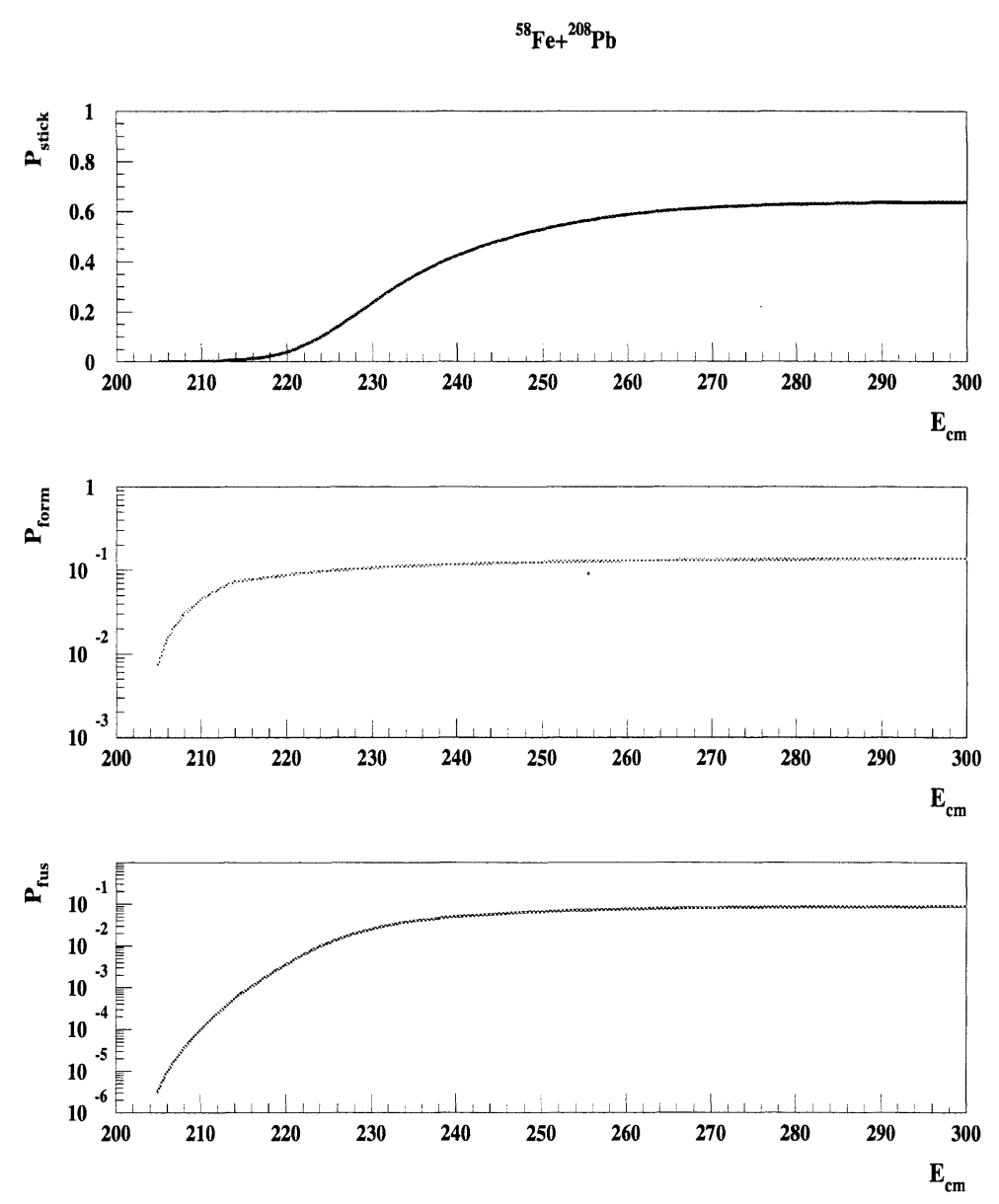

Figure 2: Calculated probabilities for the sticking, the formation and the fusion are shown for the case of ${ }^{58} \mathrm{Fe}+{ }^{208} \mathrm{~Pb}$ with $J=0$

probability(shown in the bottom) is very small in low energies due to both factors and is less than $1 / 10$ even in the higher energy limit, mainly due to the formation probability.

Next, we calculate the fusion excitation function to compare with the data available. The cross section is defined as usual,

$$
\sigma_{\text {fusion }}\left(E_{c . m .}\right)=\pi \cdot \lambda^{2} \Sigma_{J}(2 J+1) \cdot P_{\text {fusion }}^{J}\left(E_{c . m .}\right)
$$

In Fig. 3, the comparison is made on ${ }^{58} \mathrm{Fe}+{ }^{208} \mathrm{~Pb}$ system. It is apparently seen that the present model reproduces the experiment [19] almost perfectly. It is surprising, because there is no adjustable parameter in the present calculations. It is worth to remind that as the data typically show the hindrance, the present calculations precisely reproduce the hindered fusion excitation. The other systems of the so-called cold fusion path are also calculated, but there is no data to be compared for the moment. Precise measurements of the excitations of these systems are being waited for. As for the systems of the so-called hot fusion paths, the comparisons with the available data were already made successfully [5], though the reproductions are not so impressive like the above case.

In individual incident systems, there are variations due to possible effects from properties of projectiles and targets, such as deformation, shell closure, large neutron excess, etc. But the present model provides the basic values or bare values of the fusion cross sections, on which individual effects should be added for comparisons with measured data. 


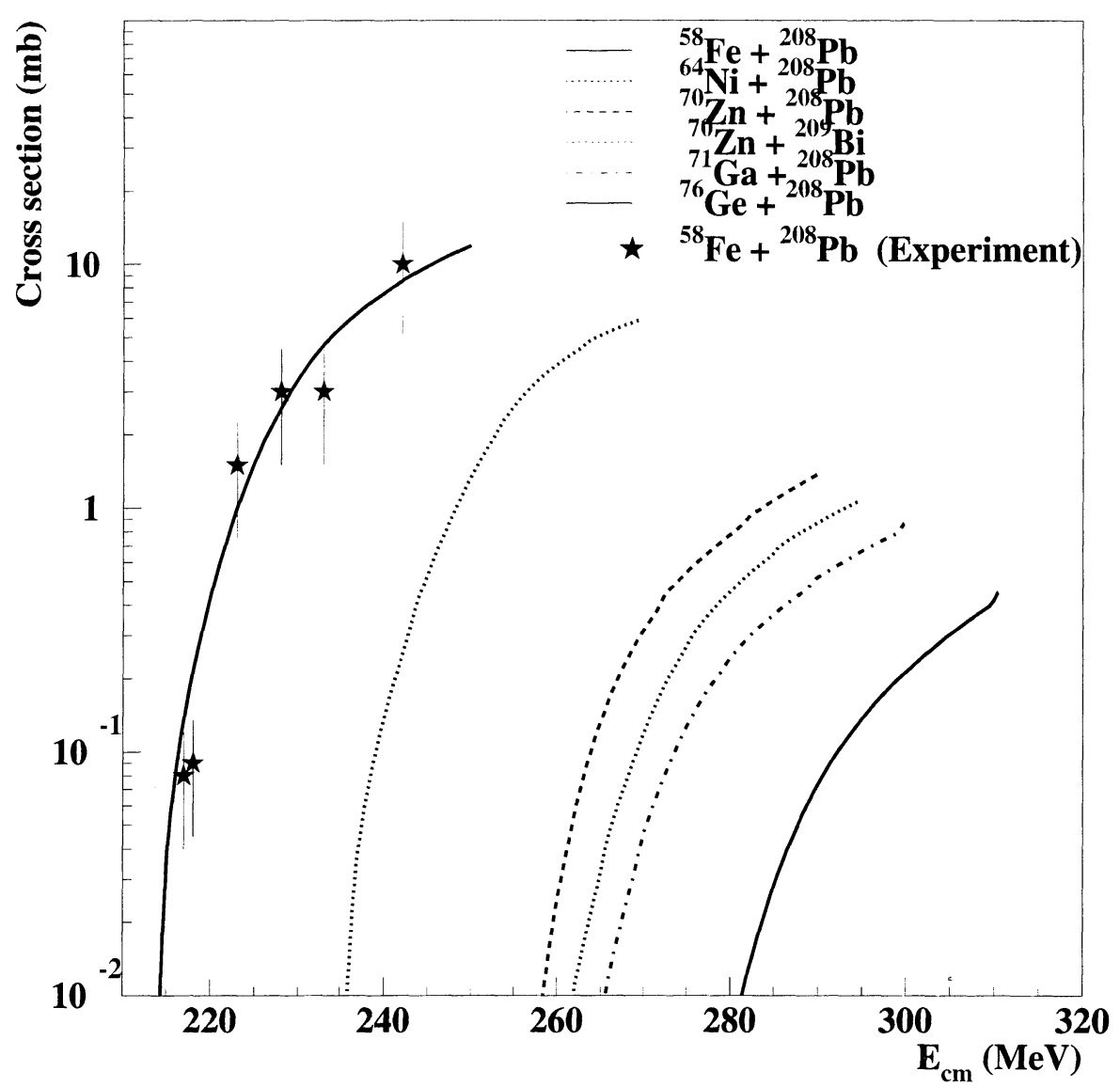

Figure 3: Calculated fusion excitation functions are shown for several systems of the cold fusion path. Experimental data are available only for ${ }^{58} \mathrm{Fe}+{ }^{208} \mathrm{~Pb}$ system, which are seen to be reproduced almost perfectly.

\subsection{Residue cross sections for the superheavy elements}

Combined with the statistical code for the survival probability [20], we calculate residue cross sections for several systems of the cold fusion path. Since masses are not known in the superheavy element region, we have to rely upon predictions by structure calculations. We, thus, adopt the mass table [21] as a reference. Fig. 4 shows comparisons of calculated $1 n$ residue cross sections with the measured data [22]. It is seen that the peak positions are well reproduced, considering the ambiguities in experimental values of $\mathrm{E}_{c . m}$. The absolute values of the peaks, however, are not reproduced well. The theoretical calculations always give much larger values, more than one order of magnitude. The values presented in the figure are obtained by the use of reduced shell correction energies by the factor is about $1 / 2$. This factor is only one free parameter introduced in the present calculations. Of course, we could introduce another way of reducing the cross sections, for example, could take a smaller value of the so-called shell damping energy which is taken to be $18 \mathrm{MeV}$ in the present calculations according to Ignatyuk [23]. Anyhow, the successful reproduction of a series of the data with the only one common parameter can be considered to be a canonical step toward a construction of a theory for the synthesis of the superheavy elements.

With the same procedure, we calculated the heavier systems not yet measured. The results are presented in Fig. 5. Recently, RIKEN has measured ${ }^{70} \mathrm{Zn}+{ }^{209} \mathrm{Bi}$, whose cross section is estimated to be smaller than $0.1 \mathrm{pb}[24]$, so the theoretical prediction appears to be too large, though the experiment is still being continued.

As for the hot fusion path, the present theory also gives the results of $x n$ cross sections 

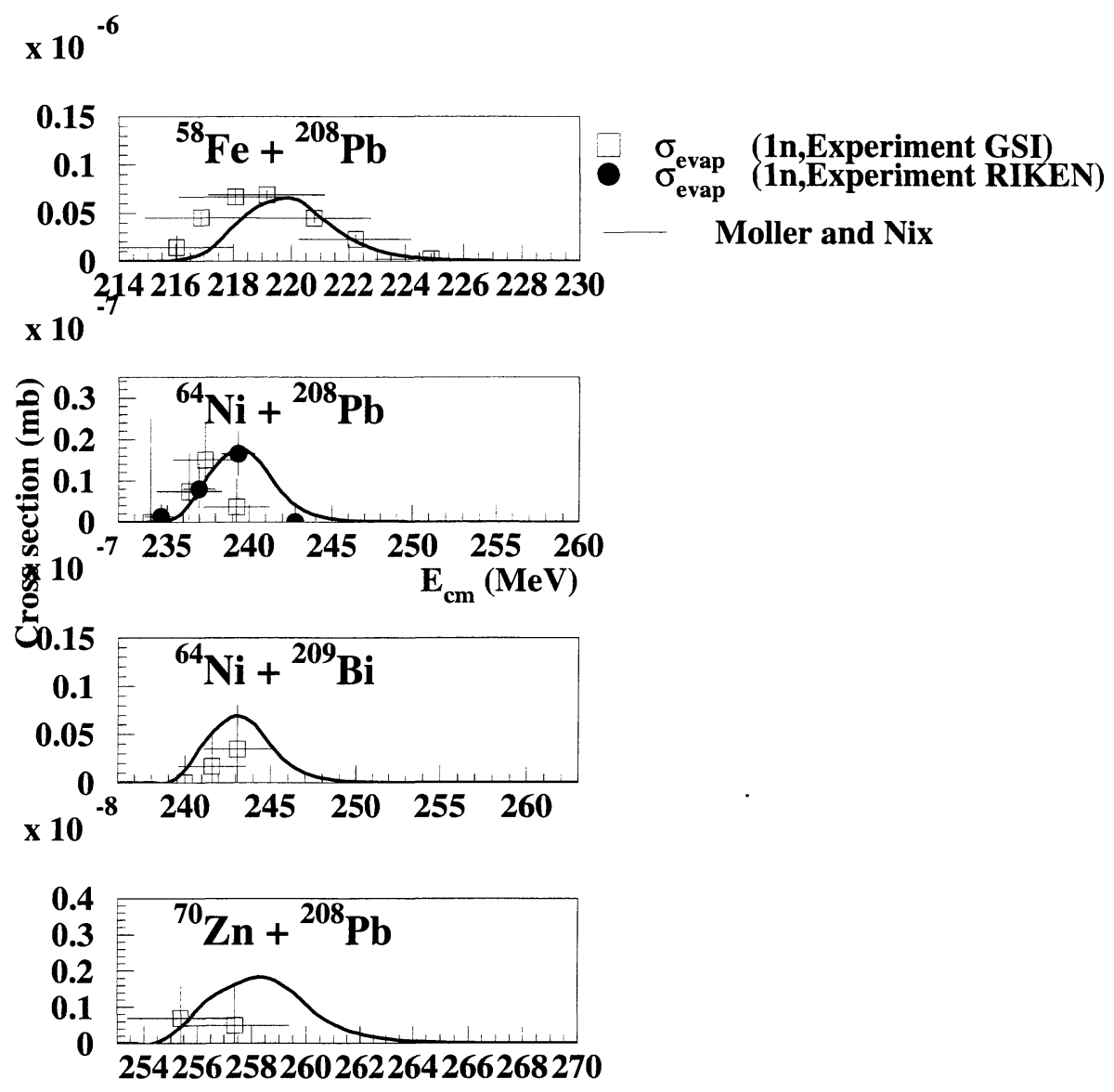

Figure 4: Comparisons are given for several systems of the cold fusion path (see the text for the explanation). RIKEN data are from K. Morita, private communication.

which are consistent with the measured data [5]. There, we have to reduce the absolute values of the predicted shell correction energies also by about factor $1 / 2$ in order to reproduce the measured magnitudes [25].

\section{Toward Heavier Elements and $N=184$ Closed Shell}

In the cold fusion path, as described above, residue cross sections become smaller, and thus, syntheses become more difficult, as compound nuclei become heavier. And even if synthesized, the nuclides are located naturally in the proton-rich side. The hot fusion path can reach isotopes with a little more neutrons, but still far from $N=184$ closed shell. A possible way is to use neutron-rich isotopes as a projectile and/or a target. Nowadays, we can expect that a variety of secondary beams of neutron-rich isotopes are available or will be. As an example, we investigate ${ }^{132} \mathrm{Sn}+{ }^{160} \mathrm{Gd}$ system which leads to a compound nucleus with $Z=114$ and $N=178$, which is not equal to, but close to 184 . Since the incident system is almost mass-symmetric, an extreme hindrance is inferred. With the present model, we can investigate quantitatively how strongly hindered it is and how much the residue cross sections are. In Fig. 6, calculated fusion excitation function is shown, which shows very small cross sections, as expected. But, it should be mentioned that the present calculations give base-line results. That is, the present calculations do not take into account possible effects of anomalous enhancements which are already observed in ${ }^{132} \mathrm{Sn}+{ }^{64} \mathrm{Ni}$ system [26]. Although an underlining mechanism is not yet clarified, it is speculated due to excess neutrons in ${ }^{132} \mathrm{Sn}$. Thus, the same effects would be expected in 

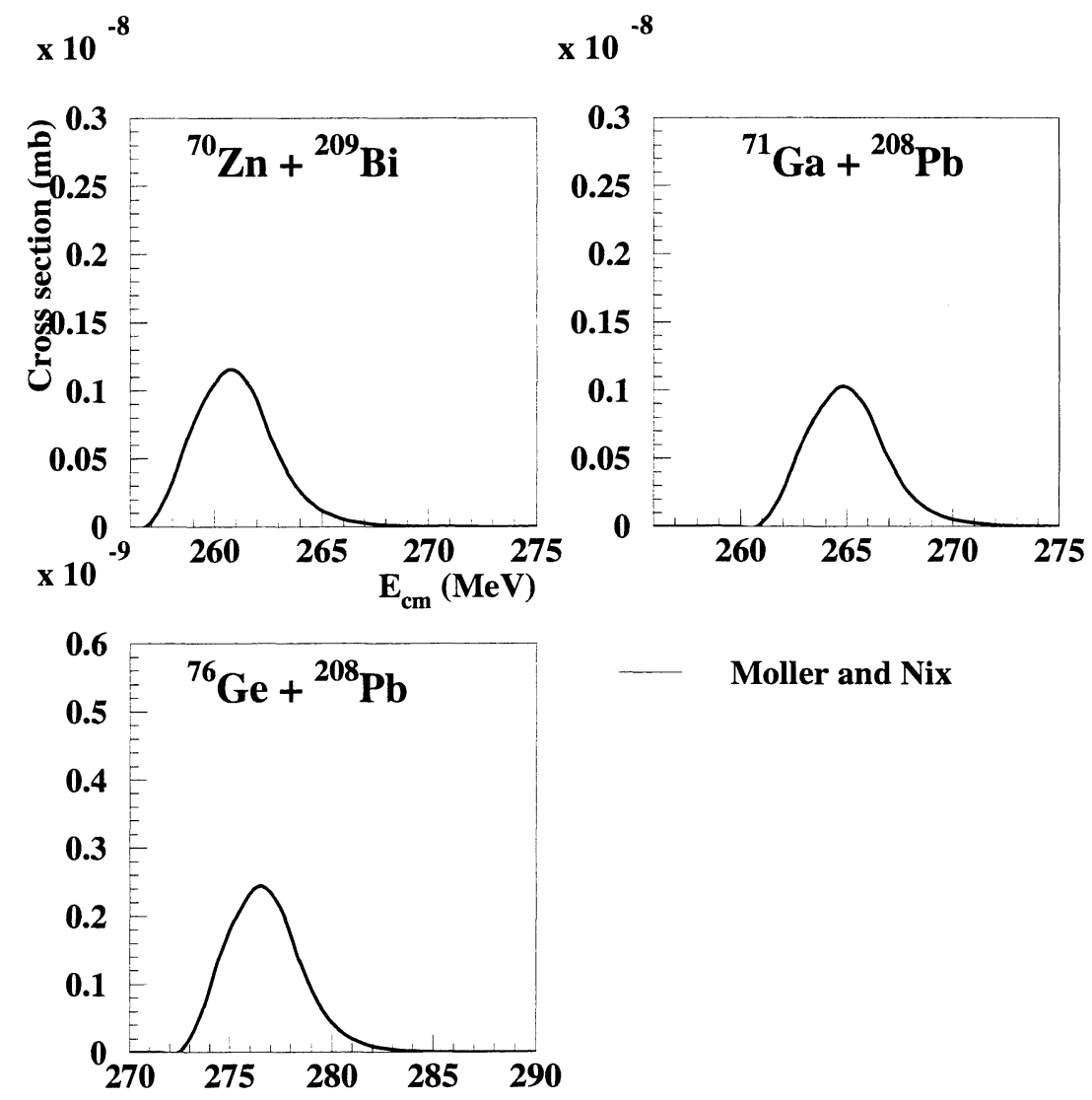

\section{Moller and Nix}

Figure 5: Predictions are given for excitation functions of $1 \mathrm{n}$ cross sections for $Z=113$ and 114 of the cold fusion path.

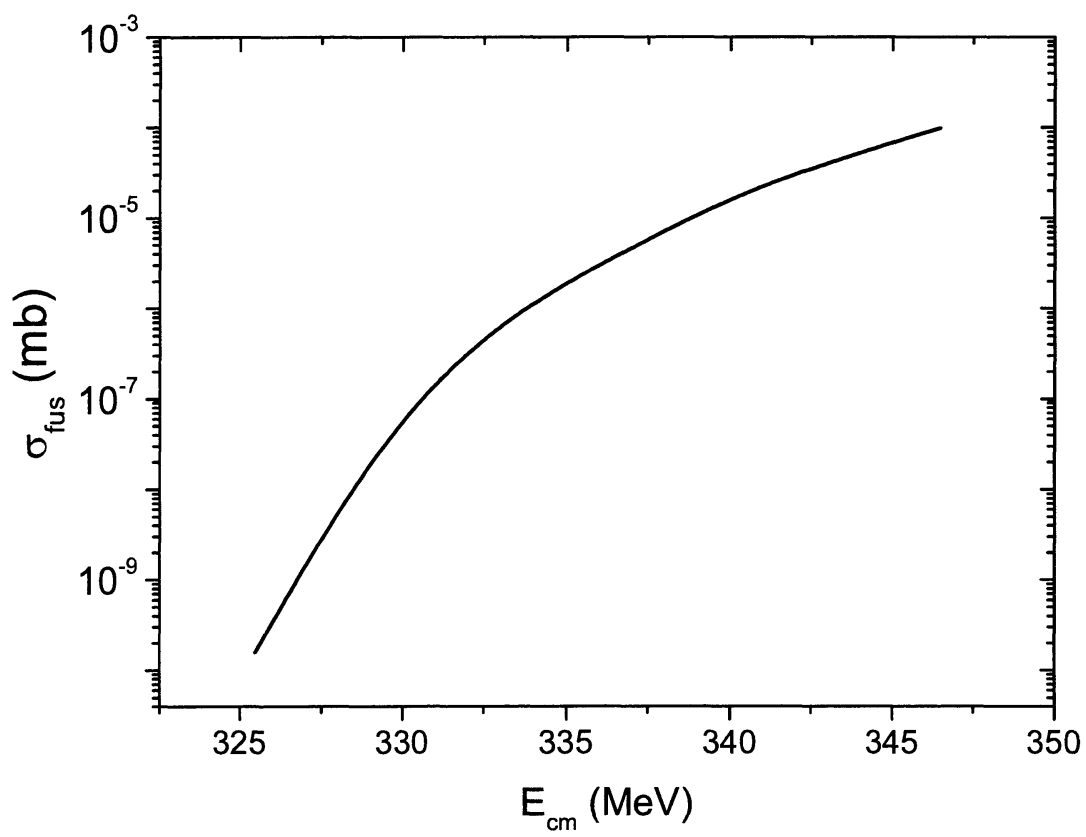

Figure 6: Fusion cross sections for ${ }^{132} \mathrm{Sn}+{ }^{160} \mathrm{Gd}$ systems by the two-step model, without taking into account anomalous enhancements expected. see the text. 


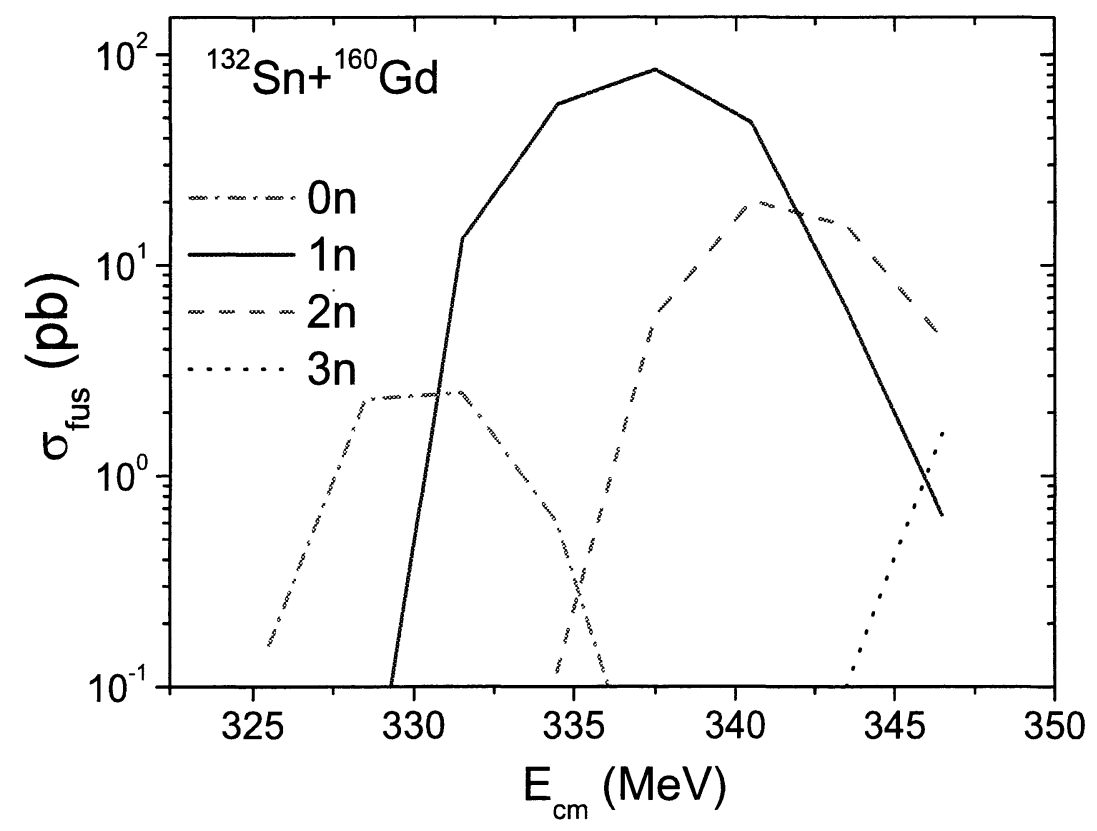

Figure 7: Preliminary results for $x n$ cross sections for ${ }^{132} \mathrm{Sn}+{ }^{160} \mathrm{Gd}$ systems. The fusion hindrance is taken into account in the fusion probability, while the anomalous enhancement is not, though it is expected due to the excess neutrons. The latter would give an additional factor more than one order of magnitude.

${ }^{132} \mathrm{Sn}+{ }^{160} \mathrm{Gd}$ system. If so, actual cross sections would be expected to be larger than the calculated values shown in Fig. 6 by an order of magnitude in low energies. For the moment, without taking into account of the effects, we calculate residue cross sections for $x n$ reactions, combining with the survival probability calculated with the statistical code. Fig. 7 shows preliminary results of excitation functions for $x n$ reactions, again using the reduction factor $1 / 2$ of shell correction energies. Since a beam intensity of secondary beam is much smaller than stable beams, it seems to be difficult to observe the residues practically. But considering the additional fusion enhancement mentioned above and expected innovations in the intensity of secondary beams, it is worthwhile to investigate the possibility to use exotic isotopes to reach the superheavy island predicted so long.

One of the author(Y.A.) acknowledges suggestions by T. Nomura on new possible systems with the use of neutron-rich beams such as ${ }^{132} \mathrm{Sn}$, and also supports by Huzhou Univ. for his visit to the Univ., where he, together with another author(C.W.S.), completed the present manuscript.

1. P.Armbruster//Annu.Rev.Nucl.Part.Sci.2000.V50.P.411.

2. S.J.Nilsson et al.//Nucl.Phys.1969.V.A131.P.1.

Z.Patyk and A.Sobiczewski//Nucl.Phys.1991.V.A533.P.132.

P.Moeller and R.Nix//J.Phys.1994.V.G20.P.1681.

P.G.Reinhard and H.Flocard//Nucl.Phys.1995.V.A584.P.467.

S.Cwiok et al.//Nucl.Phys.1996.V.A611.P.211. 
R.Smolanczuk//Phys.Rev.1999.V.C56.P.812.

M.Bender et al.//Eur.Phys.J.2000.V.A7.P.467.

3. V.M.Strutinski//Nucl.Phys.1968.V.A95.P.420;1968.V.A122.P.1.

4. W.Reisdorf//J.Phys.1982.V.G20.P.1297.

K.-H.Schmidt and W.Morawek//Rep.Prog.Phys.1991.V.54.P.949.

A.B.Quint et al.//Z.Phys.1993.V.A346.P.199.

5. Y.Abe et al.//Prog.Theor.Phys.Suppl.2002.V.146.P.104.

Y.Abe et al.// Phys.Atomic Nuclei.2003.V.66.P.1057.

6. Y.Abe and B.Bouriquet//Acta Physica Polonica.2003.V.B34.P.1927.

7. HIVAP, private communication with W.Reisdorf

KEWPIE,B.Bouriquet,D.Boilley and Y.Abe//Comp.Phys.Comm.2004.V.159.P.1.

8. Y.Abe//Eur.Phys.J.2002.V.A13.P.143.

9. D.H.E.Gross and H.Kalinowski//Phys.Rept.1978.V.45.P.175.

10. A.B.Balantekin and N.Takigawa//Rev.Mod.Phys.1998.V.70.P.77.

11. D.H.E.Gross and H.Kalinowski//Phys.Lett.1974.V.48B.P.302,and Ref.9.

12. Y.Abe,C.Gregoire and H.Delagrange//J.Phys.1986.V.47.P.C4-329.

Y.Abe et al.//Phys.Rept.1996.V.275.Nos 2.and 3.

13. G.Kosenko,C.Shen and Y.Abe//J.Nucl.Radiochem.Sci.2002.V.3.P.19.

14. J.Blocki et al.//Ann.Phys.(NY)1978.V.113.P.330.

15. T.Wada,Y.Abe and N.Carjan//Phys.Rev.Lett.1993.V.70.P.3538.

16. Y.Abe et al.//Phys.Rev.2000.V.E61.P.1125.

Y.Abe et al.//Acta Physica Polonica.2003.V.B34.P.2091.

D.Boilley,Y.Abe and J.Bao//Eur.Phys.J.2003.V.A18.P.627.

17. W.J.Swiatecki et al.//Acta Physica Polonica.2003.V.B34.P.2049.

18. B.Bouriquet,G.Kosenko and Y.Abe//Prog.Theor.Phys.Suppl.2004.V.154.P.425;

Eur.Phys.J.2004.V.A22.P.9.

19. M.G.Itkis et al.//Int.Workshop on Fusion Dynamics at the Extremes,Dubna,2527,May,2000 (World Scientific Inc.2001)P.93.

20. KEWPIE in Ref.7,revised by A. Marchix.

21. P.Moeller et al.//Atomic Data and Nuclear Data Tables.1995.V.59.P.185.

22. S.Hofmann et al.//Rep.Prog.Phys.1998.V.61.P.639.

S.Hofmann and G.Muenzenberg//Rev.Mod.Phys.2000.V.72.P.733.

23. A.V.Ignatyuk et al.//Sov.J.Nucl.Phys.1975.V.21.P.255. 
24. K.Morita et al.//J.Phys.Soc. 2004.V.73.p.2593.

25. Y.Abe et al.//to be published in Proc.EXON2005,June,2004,St.Petersburg,Russia (World Scientific Inc.2005).

26. J.F.Liang et al.//Phys.Rev.Lett.2003.V.91.P.152701. 